\title{
Critical role of galectin-3 in phagocytosis by macrophages
}

\author{
Hideki Sano, ${ }^{1}$ Daniel K. Hsu, ${ }^{1,2}$ John R. Apgar, ${ }^{3}$ Lan Yu,,${ }^{1,2}$ Bhavya B. Sharma, ${ }^{1}$ \\ Ichiro Kuwabara, ${ }^{1,2}$ Shozo Izui, ${ }^{4}$ and Fu-Tong Liu ${ }^{1,2}$ \\ ${ }^{1}$ Division of Allergy, La Jolla Institute for Allergy and Immunology, San Diego, California, USA \\ ${ }^{2}$ Department of Dermatology, University of California, Davis, School of Medicine, Sacramento, California, USA \\ ${ }^{3} \mathrm{BD}$ Pharmingen, La Jolla, California, USA \\ ${ }^{4}$ Department of Pathology, University of Geneva, Geneva, Switzerland
}

Galectin-3 is a member of a large family of animal lectins. This protein is expressed abundantly by macrophages, but its function in this cell type is not well understood. We have studied the effect of galectin-3 gene targeting on phagocytosis, a major function of macrophages. Compared with wild-type macrophages, galectin-3-deficient ( $\left.\mathrm{gal3}^{-{ }^{-}}\right)$cells exhibited reduced phagocytosis of IgGopsonized erythrocytes and apoptotic thymocytes in vitro. In addition, $\mathrm{gal}^{-/-}$mice showed attenuated phagocytic clearance of apoptotic thymocytes by peritoneal macrophages in vivo. These mice also exhibited reduced IgG-mediated phagocytosis of erythrocytes by Kupffer cells in a murine model of autoimmune hemolytic anemia. Additional experiments indicate that extracellular galectin-3 does not contribute appreciably to the phagocytosis-promoting function of this protein. Confocal microscopic analysis of macrophages containing phagocytosed erythrocytes revealed localization of galectin-3 in phagocytic cups and phagosomes. Furthermore, gal3 ${ }^{-/-}$ macrophages exhibited a lower degree of actin rearrangement upon Fc $\gamma$ receptor crosslinkage. These results indicate that galectin-3 contributes to macrophage phagocytosis through an intracellular mechanism. Thus, galectin-3 may play an important role in both innate and adaptive immunity by contributing to phagocytic clearance of microorganisms and apoptotic cells.

J. Clin. Invest. 112:389-397 (2003). doi:10.1172/JCI200317592.

\begin{abstract}
Introduction
Macrophages play an important role in innate and adaptive immunity as professional phagocytes by internalizing and degrading pathogens $(1,2)$. They recognize pathogens that are opsonized by specific Ab's, through specific receptors, such as Fcy receptors (Fc $\gamma$ R's) and complement receptor-3 (CR3; also known as Mac-1 or CD11b/18 integrin). Ligation of these receptors is accompanied by activation of specific signaling pathways and cytoskeletal changes, such as filamentous actin (F-actin) redistribution, as well as production of superoxide anion, proinflammatory cytokines, and chemokines such as TNF- $\alpha$, IL-1, and IL-8 (2). Macrophages also play an important role in a variety of physiological and pathologi-
\end{abstract}

Received for publication December 11, 2002, and accepted in revised form May 20, 2003.

Address correspondence to: Fu-Tong Liu, Department of Dermatology, University of California, Davis, School of Medicine, 4860 Y Street, Suite 3400, Sacramento, California 95817, USA. Phone: (916) 734-6795; Fax: (916) 734-6793; E-mail: fliu@ucdavis.edu.

Hideki Sano's present address is: Sakura Hospital, Toho University, Sakura-city, Chiba, Japan.

Hideki Sano and Daniel K. Hsu contributed equally to this work.

Conflict of interest: The authors have declared that no conflict of interest exists.

Nonstandard abbreviations used: $\mathrm{F}_{c} \gamma$ receptor $(\mathrm{Fc} \gamma \mathrm{R})$; filamentous actin (F-actin); galectin-3-deficient (gal3 $\left.3^{--}\right)$; sheep red blood cell (srbc); bone marrow-derived macrophage (BMM $\Phi)$. cal processes by phagocytosing apoptotic cells. They recognize these cells by using other receptors, including class A and B scavenger receptors, CD36, and CD14 (2). This process is accompanied by production of anti-inflammatory cytokines and mediators such as TGF- $\beta$, IL- 10 , and $\mathrm{PGE}_{2}$ (2).

Galectin- 3 is a member of large family of $\beta$-galactoside-binding animal lectins (3). It is secreted by various cell types including monocytes, macrophages, and epithelial cells $(4,5)$. The released protein can function as an extracellular molecule to activate cells (6-11), mediate cell-cell and cell-ECM interactions (12-14), and induce migration of monocytes, macrophages (15), and endothelial cells (16), probably by binding to pertinent receptors through lectin-carbohydrate interactions. More recently, cell surface galectin-3 was implicated in restricting $\mathrm{T}$ cell receptor clustering and thus negatively regulating $\mathrm{T}$ cell activation (17). This protein is also abundantly present inside the cells and has been shown to play important roles in some biological responses through its intracellular actions (18). For example, it has been identified as a factor in premRNA splicing (19) and as a regulator of the cell cycle (20) and apoptosis $(21,22)$, although the precise mechanisms of action are still undetermined.

Galectin-3 expression is highly upregulated when monocytes differentiate into macrophages (6) and are downregulated when these cells differentiate into dendritic cells (23), suggesting that this protein might have functions specifically associated with particular differentiation stages in the monocyte cell lineage. The 
recent findings that galectin-3 is a major component of phagosomes in a mouse macrophage cell line (24) and of exosomes derived from a dendritic cell line (25) further suggest that this protein may be involved in endocytosis and antigen presentation. Here, we have studied the role of endogenous galectin- 3 in the phagocytic function of macrophages by using galectin-3deficient $\left(\mathrm{gal3}^{--}\right)$mice. We found that galectin-3 is essential for effective phagocytosis of both IgGopsonized erythrocytes and apoptotic cells in vitro and in vivo. It participates in the phagocytic process probably through an intracellular mechanism.

\section{Methods}

Mice and reagents. Gal3 ${ }^{-/-}$mice were generated as described previously (26) and backcrossed to C57BL/6 mice for nine generations. Recombinant mouse GM-CSF was provided by Kirin Brewery Co. Ltd. (Maebashi, Japan). Sheep red blood cell (srbc) suspension was obtained from ICN Biomedicals Inc. (Aurora, Ohio, USA). Goat anti-galectin-3 Ab (6) and mouse anti-mouse rbc mAb 34-3C (IgG2a) (27) have been described previously. Rabbit polyclonal anti-srbc Ab was from Sigma-Aldrich (St. Louis, Missouri, USA). Rat anti-mouse Fc $\gamma R$ (CD16/CD32) mAb 2.4G2 and FITC-conjugated annexin $V$ were obtained from $\mathrm{BD}$ Pharmingen (San Diego, California, USA). Alexa 488and FITC-conjugated mouse anti-goat IgG, TOTO-3, rhodamine-phalloidin, NBD-phallacidin, and DiD oil (1,1'-dioctadecyl-3,3,3',3'-tetramethylindodicarbocyanine perchlorate) were purchased from Molecular Probes Inc. (Eugene, Oregon, USA). Rhodamine-conjugated donkey anti-goat IgG and $\mathrm{F}\left(\mathrm{ab}^{\prime}\right)_{2}$ fragment of mouse anti-rat IgG were from Jackson ImmunoResearch Laboratories Inc. (West Grove, Pennsylvania, USA). RPMI 1640 was purchased from Invitrogen (Grand Island, New York, USA).

Preparation of monolayer cultures of macrophages. Mouse bone marrow-derived macrophages (BMM $\Phi)$ were prepared as adherent cultures using previously described procedures (28), except that recombinant mouse GM-CSF $(10 \mathrm{ng} / \mathrm{ml})$ was used instead of L-cell-conditioned medium. More than $95 \%$ of the cells from both wild-type and gal3 $3^{--}$mice showed the characteristic appearance of macrophages when examined by light microscopy following Wright staining. Preparation of IgG-opsonized srbc's and apoptotic thymocytes. Opsonization of srbc's was performed by treating the cells with a subagglutinating concentration of antisrbc $\mathrm{Ab}$ for 30 minutes at $37^{\circ} \mathrm{C}$ with gentle agitation. Thymocytes were harvested from the thymus of 3- to 6week old mice. Apoptosis was induced by treating these cells with $1 \mu \mathrm{g} / \mathrm{ml}$ of dexamethasone in 10\% FBS/RPMI 1640 for 4 hours at $37^{\circ} \mathrm{C}(29)$. Fifty to sixty percent of the cells of the resulting population was in early apoptosis, as confirmed by flow cytometric analysis following FITC/annexin $\mathrm{V}$ and propidium iodide staining.

In vitro phagocytosis assays. Synchronous phagocytosis assays were performed according to a previously described method (28). In brief, opsonized srbc's or apoptotic thymocytes were washed with PBS twice and added to semiconfluent cultured BMM $\Phi$ in 12-well microtiter plates; the plates were then placed on ice for 15 minutes. Afterward, the plates were centrifuged at $150 \mathrm{~g}$ for 5 minutes at $4^{\circ} \mathrm{C}$ to promote contact between the added cells and macrophages. Phagocytosis was allowed to occur by replacing the supernatant with medium prewarmed to $37^{\circ} \mathrm{C}$ and placing the plates in a $5 \% \mathrm{CO}_{2}$ incubator at $37^{\circ} \mathrm{C}$. After the indicated time periods, the phagocytic reaction was stopped by adding an equal volume of $4 \%$ formaldehyde/PBS. The cell mixtures were washed with cold PBS followed by ACK lysing buffer $\left(0.15 \mathrm{M} \mathrm{NH}_{4} \mathrm{Cl}\right.$, $1.0 \mathrm{M} \mathrm{KHCO}_{3}$, and $0.01 \mathrm{M} \mathrm{Na}_{2}$ EDTA, pH 7.2) to remove unphagocytosed srbc's and then fixed with $4 \%$ formaldehyde/PBS. Digital images of randomly selected fields under phase-contrast microscopy were captured and the number of engulfed cells in more than 300 macrophages was counted. To measure phagocytosis of apoptotic thymocytes, macrophages were further processed for TUNEL staining and phagocytosed thymocytes were detected by confocal fluorescence microscopy as described below under Cell staining and confocal microscopy. The phagocytic index was calculated according to the following formula: phagocytic index $=($ total number of engulfed cells $/$ total number of counted macrophages $) \times$ (number of macrophages containing engulfed cells/total number of counted macrophages) $\times 100$.

Unsynchronized phagocytosis assays were performed as described above except that mixtures of macrophages and srbc's were not cooled to $4^{\circ} \mathrm{C}$ before initiation of phagocytosis. Thus, BMM $\Phi$ were cultured in 12-well plates on glass coverslips to subconfluence. Cells were removed from the $\mathrm{CO}_{2}$ incubator, and opsonized srbc's in prewarmed medium at $37^{\circ} \mathrm{C}$ were added to the macrophages. Following centrifugation at $400 \mathrm{~g}$ for 1 minute at room temperature, the plates were returned to the $\mathrm{CO}_{2}$ incubator at $37^{\circ} \mathrm{C}$. Phagocytosis was stopped 20 minutes after addition of opsonized srbc's to macrophages as described above for synchronous assays. After fixation, coverslips were mounted on slides, engulfed srbc's were counted under phase-contrast microscopy, and phagocytic index was calculated as above.

Phagocytosis in cocultures of wild-type and gal3 $3^{-/-}$ macrophages was evaluated with a mixture of approximately equal numbers of both genotypes of BMM $\Phi$ that had been cultured in the same well for 2-5 days. The phagocytosis assay using opsonized srbc's was conducted as described above in synchronized assays. After the indicated period, the wild-type and gal3-/cells were differentiated by immunostaining (see Cell staining and confocal microscopy, below) using anti-galectin-3 $\mathrm{Ab}$ and chromogenic detection with an alkaline phosphatase-tagged secondary $\mathrm{Ab}$. The phagocytic index for each macrophage population was determined as described above. 
Alternatively, fluorescence-labeled cells were used as the target cells and phagocytosis was measured by flow cytometric analysis. Briefly, srbc's were labeled with FITC-albumin or DiD cell labeling solution at the same time of opsonization at $37^{\circ} \mathrm{C}$ for $30 \mathrm{~min}$ utes. The labeled cells were washed twice with PBS and applied to the assay plates as described above. Afterward, the wells were washed with PBS and the adherent macrophages were removed from the plates by treatment with trypsin-EDTA and then examined by flow cytometry using a FACScan cytometer (Becton, Dickinson and Co., Franklin Lakes, New Jersey, USA). A micropinocytosis assay was performed by incubating cultured macrophages with FITC-conjugated dextran ( $\mathrm{mol} \mathrm{wt}, 12,000$ ), with or without activation by PMA. The cells were then harvested and analyzed by flow cytometry as described above.

In vivo phagocytosis of apoptotic cells. The in vivo phagocytic clearance of apoptotic cells was examined according to a previously described method (29). Briefly, mice were injected intraperitoneally with $1.5 \mathrm{ml}$ of sterile $3 \%$ Brewer's thioglycollate to induce peritonitis. Four days later, $2 \times 10^{7}$ apoptotic thymocytes prepared as described above in $200 \mu \mathrm{l}$ PBS were injected into the peritoneum. After 30 minutes, mice were sacrificed and the peritoneal cells were collected by lavaging the cavity with ice-cold PBS containing $5 \mathrm{mM}$ EDTA. The cells were spun onto glass slides and examined by light microscopy following Wright staining. Macrophages containing internalized apoptotic thymocytes and/or apoptotic bodies were enumerated. Thymocytes were counted as phagocytosed when more than $50 \%$ of the cell was inside the cell surface of the macrophages.

In vivo phagocytosis of IgG-opsonized cells. An experimental autoimmune hemolytic anemia model described previously $(30,31)$ was used for assessing phagocytosis in vivo. In brief, $100 \mu \mathrm{g}$ of the anti-mouse rbc $\mathrm{mAb} 34-3 \mathrm{C}$ was injected intraperitoneally into 2 -monthold mice. Three days later, blood samples, liver, and spleen were harvested from the treated animals. The degree of anemia was assessed by measuring hematocrit. Livers were processed and stained with $\mathrm{H} \& \mathrm{E}$ to assess in vivo rbc phagocytosis by resident Kupffer cells, as described $(30,31)$.

$F c \gamma R$ crosslinkage. BMM $\Phi$ were incubated in $0.1 \%$ FBS/RPMI 1640 without GM-CSF for 1-2 hours at $37^{\circ} \mathrm{C}$ and then harvested from the plates using trypsinEDTA. The cell suspension $\left(5 \times 10^{6}\right.$ cells $\left./ \mathrm{ml}\right)$ was incubated with $10 \mu \mathrm{g} / \mathrm{ml}$ rat anti-mouse Fc $\gamma R$ mAb 2.4G2 for 30 minutes at $4{ }^{\circ} \mathrm{C}$ with occasional shaking. After the cells were washed with ice-cold medium, the $\mathrm{F}\left(\mathrm{ab}^{\prime}\right)_{2}$ fragment of mouse anti-rat IgG Ab was added (final concentration, $10 \mu \mathrm{g} / \mathrm{ml}$ ) and the mixture was incubated at $37^{\circ} \mathrm{C}$ for the indicated time periods.

Cell staining and confocal microscopy. Macrophages cultured on coverslips were used in the phagocytosis assay or stimulated by Fc $\gamma \mathrm{R}$ crosslinkage as described above. After fixation with $4 \%$ paraformaldehyde/PBS at $4{ }^{\circ} \mathrm{C}$ for 12-16 hours, the cells were permeabilized with
$0.2 \%$ Triton X-100/PBS for 5 minutes on ice and incubated with goat anti-galectin-3 $\mathrm{Ab}$ followed by rabbit anti-goat Ab conjugated to FITC or Alexa 488. To detect apoptotic thymocytes phagocytosed by macrophages, TUNEL staining was performed with a commercial kit (Promega Corp., Madison, Wisconsin, USA). Subsequently, the cells were incubated with propidium iodide or TOTO-3 (for nucleic acid staining) or rhodamine-phalloidin (for F-actin staining). After the coverslips were mounted on the glass slides, digital images from a confocal fluorescence microscope were captured in two or three channels in an MRC1024 Laser Scanning Confocal Image System (Bio-Rad Laboratories Inc., Hercules, California, USA).

Quantitative measurement of F-actin. The total F-actin content in macrophages was measured as described previously (32). Briefly, after macrophages were treated with specific Ab's for indicated time periods to crosslink Fc $\gamma \mathrm{R}$, formaldehyde (3.7\% final, vol/vol) was added to stop the reaction. The fixed cells were permeabilized with $10 \mathrm{mM}$ imidazole, $40 \mathrm{mM} \mathrm{KCl}, 10$ mM EGTA, $1 \mathrm{mM} \mathrm{MgCl}_{2}$, and $1 \%$ Triton X-100. F-actin was then stained with NBD-phallacidin for 1 hour at room temperature. After the cells were washed with PBS, F-actin-bound NBD-phallacidin was extracted with methanol. The extracts were centrifuged to remove any insoluble material, and relative fluorescence was measured using an AMINCO Bowman Series 2 spectrofluorometer (Thermo Spectronic, Madison, Wisconsin, USA) with the excitation and emission wavelengths set at $465 \mathrm{~nm}$ and $535 \mathrm{~nm}$, respectively. The F-actin ratio was calculated as (F-actin in activated cells - background)/(F-actin in unstimulated cells - background).

Data analysis. Data are presented as mean \pm SD unless otherwise stated. The statistical examination of results was performed by ANOVA using Fisher's protected least significant difference test for multiple comparisons. Analysis of the results from the in vivo experiments of experimental autoimmune hemolytic anemia and phagocytic clearance of apoptotic thymocytes was accomplished by the Mann-Whitney $U$ test. $P$ values below 0.05 were considered significant.

\section{Results}

Gal3 ${ }^{-/-}$macrophages exhibit delayed phagocytosis of IgGopsonized erythrocytes in vitro. We conducted in vitro phagocytosis assays with BMMФ from wild-type and gal3 $3^{-/-}$mice to analyze the role of galectin-3 in Fc $\gamma \mathrm{R}-$ mediated phagocytosis. We used a previously described method that allows phagocytosis by all macrophages in the culture to be synchronized (28). As shown in Figure 1a, wild-type BMM $\Phi$ engulfed IgG-opsonized srbc's within 5 minutes, consistent with previous studies (28). In contrast, gal3-/BMM $\Phi$ ingested many fewer erythrocytes during the same time period. However, the degree of phagocytosis by the two genotypes appeared to be comparable after 60 minutes (Figure 1a). 
To quantify phagocytosis, we counted the internalized cells in more than 300 macrophages at different timepoints and calculated the phagocytic index. Figure $1 \mathrm{~b}$ shows that phagocytosis of srbc's by wild-type macrophages was nearly completed at the earliest timepoint tested (10 minutes); the phagocytic index reached a maximum after 20 minutes. In contrast, the phagocytic index of gal3 $3^{-/-}$macrophages was lower than that of the wild type by $59.4 \%$ and $51.4 \%$ at 10 minutes and 20 minutes, respectively $(n=5$, $P<0.001$, Figure 1b). Phagocytosis then continued to increase and became comparable to that of the wild type. The impaired FcyR-mediated response in these cells is not due to the reduced quantity of Fc $\gamma R$, because the level of cell surface Fc $\gamma \mathrm{R}$ is comparable between wild-type and gal3 $3^{-/-}$macrophages, as determined by flow cytometry (data not shown). It is also not due to reduced capacity to bind opsonized srbc's, as no differences in numbers of bound srbc's were detected on macrophages at $4{ }^{\circ} \mathrm{C}$ prior to initiation of phagocytosis by warming (data not shown). We also noted that cells of both genotypes showed a minimum rate of apoptosis ( $<1 \%$ of cells undergoing apoptosis as detected by TUNEL assay during the phagocytosis assay). Therefore, the difference in the phagocytic index is not due to a difference in cell survival between the two genotypes.

In the above assays, the mixtures of macrophages and srbc's were exposed to a low temperature $\left(4^{\circ} \mathrm{C}\right)$ before phagocytosis was initiated. In order to exclude the possibility that the defects of gal3 $3^{-/-}$ macrophages were a result of the exposure to low temperature, unsynchronized phagocytosis assays were also performed. As shown in Figure 1c, gal3-/macrophages exhibited significantly impaired phagocytosis as observed in synchronous assays. These results demonstrate that gal3 $3^{-/-}$macrophages have an intrinsic phagocytic defect.

\footnotetext{
Figure 1

Delayed phagocytosis of IgG-opsonized erythrocytes by gal3-/macrophages in vitro. (a) After opsonized srbc's were added to cultured $\mathrm{BMM} \Phi$ at $4^{\circ} \mathrm{C}$, synchronized phagocytosis was initiated by raising the temperature of the cultures to $37^{\circ} \mathrm{C}$. After the indicated time periods, unbound srbc's were removed and the cells were fixed for phase-contrast microscopy. (b) Phagocytic function was quantified by counting internalized srbc's in more than 300 macrophages and the phagocytic index was calculated. Each data point represents the mean \pm SD from five experiments. (c) Unsynchronized phagocytosis assays in $\mathrm{BMM} \Phi$ were performed at $37^{\circ} \mathrm{C}$ for 20 minutes. Data are presented as mean \pm SD from five high power fields and are representative of two experiments. $P<0.01$ by Mann-Whitney $U$ analysis between the genotypes. (d) Phagocytosis assays were performed as in a after staining of rbc's with FITCalbumin at the time of opsonization. After 30 minutes, cells were harvested, washed, and subjected to flow cytometric analysis. Results shown are representative of six experiments.
}

Delayed phagocytosis in gal3-/- macrophages was also confirmed by flow cytometric analysis. Figure 1d contains representative data from six independent experiments and shows that gal3 $3^{-/-}$macrophages internalized much smaller numbers of fluorescence-labeled srbc's than did wild-type cells after 20 minutes. These results indicate that galectin-3 is involved in the early steps of phagocytosis of IgG-opsonized particles.

In contrast to their reduced phagocytosis of IgGopsonized cells, gal3-/- macrophages displayed normal micropinocytosis of soluble substances. Flow cytometric analysis showed that wild-type and $\mathrm{gal3}^{-/-}$ macrophages, both PMA-stimulated and unstimulated, internalized almost equal amounts of FITC-labeled dextran (data not shown). The results indicate that galectin3 is involved in some but not all types of endocytosis.

a
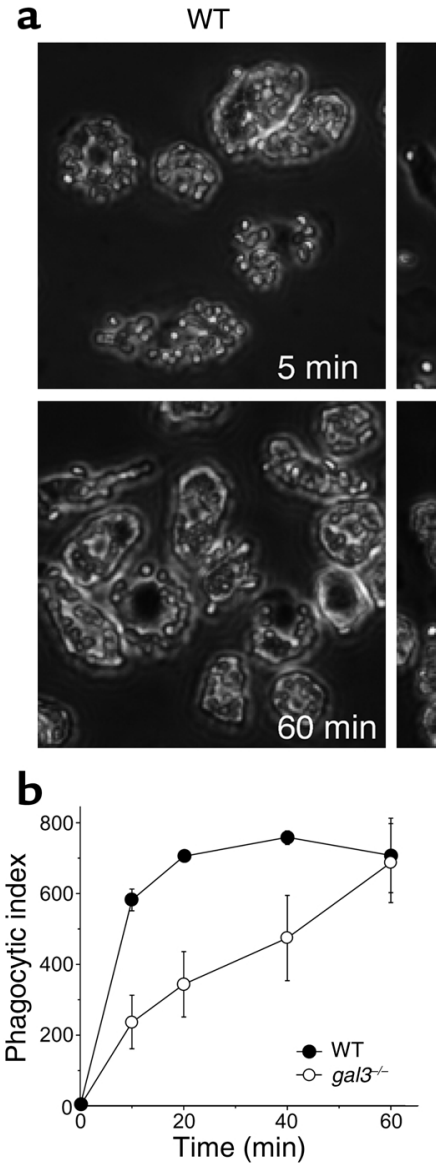

d

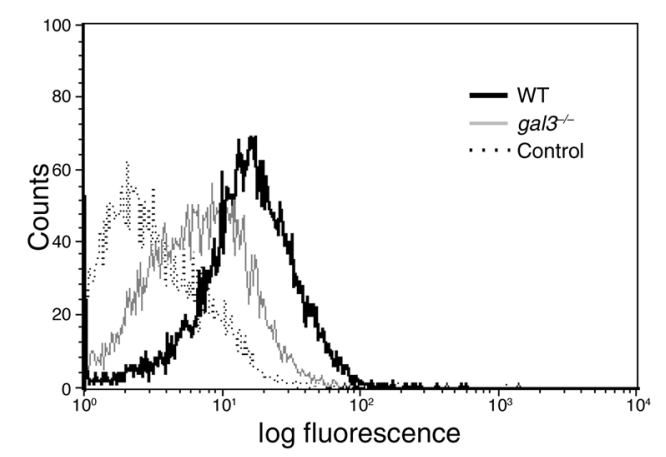



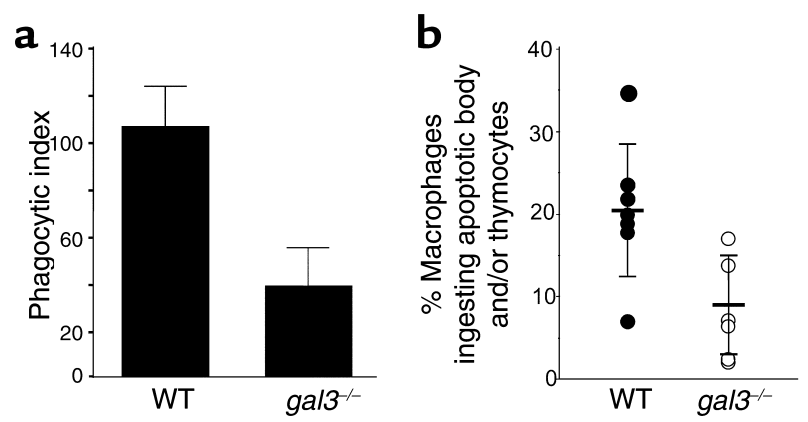

Gal3 $^{-/-}$macrophages show defective phagocytosis of apoptotic cells in vitro and in vivo. We also compared wild-type and $\mathrm{gal3}^{-/-}$macrophages for their phagocytosis of apoptotic cells. Thymocytes induced to undergo apoptosis by dexamethasone treatment were added to macrophages grown on coverslips in 12-well plates and the mixture was incubated at $37^{\circ} \mathrm{C}$. The cells were then stained by the TUNEL procedure to detect the phagocytosed apoptotic thymocytes. Figure 2 a shows that gal3 ${ }^{-/}$macrophages internalized fewer apoptotic thymocytes than did wild-type cells. The phagocytic index of $\mathrm{gal3}^{-/-}$macrophages was $64 \%$ lower than that of wild-type cells at the 20-minute timepoint (wild-type, $106 \pm 16.7$ vs. gal3 $\left.^{-/}, 38.5 \pm 16.1, n=6, P<0.001\right)$.

We then studied in vivo phagocytosis activity by injecting apoptotic thymocytes intraperitoneally into mice with thioglycollate-induced sterile peritonitis and examining the engulfment of these cells by peritoneal macrophages. As shown in Figure 2b, the percentage of macrophages that ingested apoptotic cells was lower by $57 \%$ in gal3 $^{-/-}$mice than in the wild type (wild-type, $20.7 \% \pm 8.3 \%$ vs. gal3 ${ }^{-/-}$, $9.0 \% \pm 5.6 \% ; P<0.01 ; n=7)$. These results indicate that galectin-3 plays an important role in phagocytosis of apoptotic cells.

Gal3-/- Kupffer cells in the liver show defective phagocytosis of IgG-opsonized cells in vivo. Autoimmune hemolytic anemia can be induced experimentally in mice by a single intraperitoneal injection of an IgG2a anti-mouse rbc mAb, 34-3C (30). The resultant anemia, as evaluated by a reduction in hematocrit, is primarily mediated through Fc $\gamma \mathrm{R}$-dependent erythrophagocytosis by Kupffer cells $(33,34)$. Thus, this animal model was used to determine whether galectin-3 is involved in IgG-mediated phagocytosis by Kupffer cells in vivo. We found that there was no significant difference in hematocrit between the two types of mice after injection of PBS (wild-type, $50.0 \% \pm 0.82 \%$ vs. gal3 ${ }^{-/}$, $48.8 \% \pm 0.96 \%$, $n=4)$. However, 3 days after the treatment with the anti-rbc mAb, wild-type mice showed a much more pronounced reduction in hematocrit than was seen in gal3 $^{-/-}$mice (wild-type hematocrit, $26.9 \% \pm 4.79 \%$ vs. gal3-/- hematocrit, $42.5 \% \pm 3.47 \%, P<0.005, n=4$ ) (Figure 3a). Histological examinations of the liver resected 3 days after injection of the mAb showed

\section{Figure 2}

Reduced phagocytosis of apoptotic thymocytes by gal3-/- macrophages. (a) In vitro phagocytosis of apoptotic thymocytes by macrophages was performed and the phagocytic index was calculated following 20-minute incubation of these cells. Data shown represent the mean \pm SD from six experiments $(P<0.001)$. (b) Scatter plots of the percentage of macrophages containing phagocytosed apoptotic thymocytes and/or apoptotic bodies 30 minutes after injection of the cells into the peritoneum of wild-type and gal $3^{-/}$mice with sterile peritonitis, shown as mean (heavy horizontal lines) \pm SD. Each data point represents the result from one mouse; seven mice of each genotype were used $(P<0.01)$.

that over half of the Kupffer cells in wild-type mice contained phagocytosed rbc's (Figure 3b). In contrast, only a small population of the Kupffer cells in gal3 $^{-1-}$ mice showed phagocytosis of rbc's (Figure $3 \mathrm{~b}$ ). In addition, the liver from wild-type but not $\mathrm{gal3}^{-1-}$ mice contained prominent extramedullary hematopoietic foci. These results indicate that galectin-3 is involved in erythrophagocytosis by hepatic Kupffer cells in vivo.

Extracellular galectin-3 does not contribute appreciably to phagocytosis. Galectin-3 is known to be secreted by cells, although, like other galectins, it does not contain a signal peptide. On the other hand, galectin-3 has been shown to have a variety of intracellular functions (18). As a first step in assessing whether it is intracellular or extracellular galectin-3 that is involved in the phagocytic process, we addressed whether phagocytosis of macrophages was affected by the presence of lactose in the culture media. This approach is used because all known extracellular functions of this protein in monocytes/macrophages are dependent on its C-terminal domain lectin properties, and can be inhibited by specific saccharide ligands, such as lactose $(6,15$, 35 ). We found that $5 \mathrm{mM}$ lactose did not have any effect on the degree of phagocytosis of opsonized erythrocytes by wild-type macrophages (data not shown).

To firmly establish that galectin-3 secreted from cells does not contribute to phagocytosis, we performed the phagocytosis assay with cocultures of wild-type and gal3 ${ }^{-/-}$macrophages. The two genotypes were differentiated at the end of the assay by immunostaining for galectin-3. If galectin-3 released from wild-type cells contributes to phagocytosis, it should affect both genotypes through a paracrine mechanism and the phagocytic index of $\mathrm{gal3}^{-/-}$ macrophages should approach that of the wild type. As shown in Figure 4, the phagocytic index of $\mathrm{gal3}^{-1-}$ macrophages in the cocultures remained significantly lower than that of wild-type macrophages.

Galectin-3 is localized in F-actin-rich phagocytic cups and phagosomes. To determine the galectin-3 localization in macrophages engaged in phagocytosis of IgGopsonized srbc's, we performed confocal immunofluorescence microscopic analysis. As an initial step, we confirmed the specificity of the anti-galectin-3 $\mathrm{Ab}$ used in this analysis by showing that $\mathrm{gal3}^{-/-}$ 
macrophages were not stained at all (data not shown). In resting wild-type macrophages, galectin3 distributes diffusely in the cytoplasm and showed no significant codistribution with F-actin, which was stained positively by rhodamine-phalloidin (Figure 5a). However, within 1 minute after the addition of opsonized srbc's to wild-type macrophages, galectin-3 was found in the F-actin-enriched phagocytic cups (Figure 5a). At 10 minutes, galectin-3 was found to be associated with the phagosomes, which are formed from the internalized cells and are also rich in actin (Figure 5b). After 15 minutes, galectin3 remained associated with the mature phagosomes (phagolysosomes), while F-actin was no longer present (Figure 5b). The distribution of galectin-3 in the phagolysosomes was confirmed by simultaneous staining with Ab's against galectin-3 and LAMP-1, the latter being a specific marker protein for lysosomes that is invariably present in phagosomes (data not shown). We also noted that galectin-3 is not present in the actin plaques formed at the interface between cells and culture surface both before and after phagocytosis (data not shown), indicating that this codistribution is specific for the phagocytic response. No signal for galectin- 3 was detected when nonpermeabilized cells were stained, suggesting that this protein localizes inside but not outside the plasma membrane (data not shown).

Fc $\gamma R$-mediated F-actin rearrangement is impaired in gal3-/- macrophages. Phagocytosis is known to be an actin-dependent process, and the initial step is believed to be the formation of phagocytic cups through a process that involves actin polymerization under the plasma membrane (36). Thus, we compared this response induced by Fc $\gamma \mathrm{R}$ crosslinkage in wildtype and $\mathrm{gal3}^{-/-}$macrophages by quantifying the total F-actin content in the cells. We found that Triton $\mathrm{X}-100$-insoluble F-actin content increased more

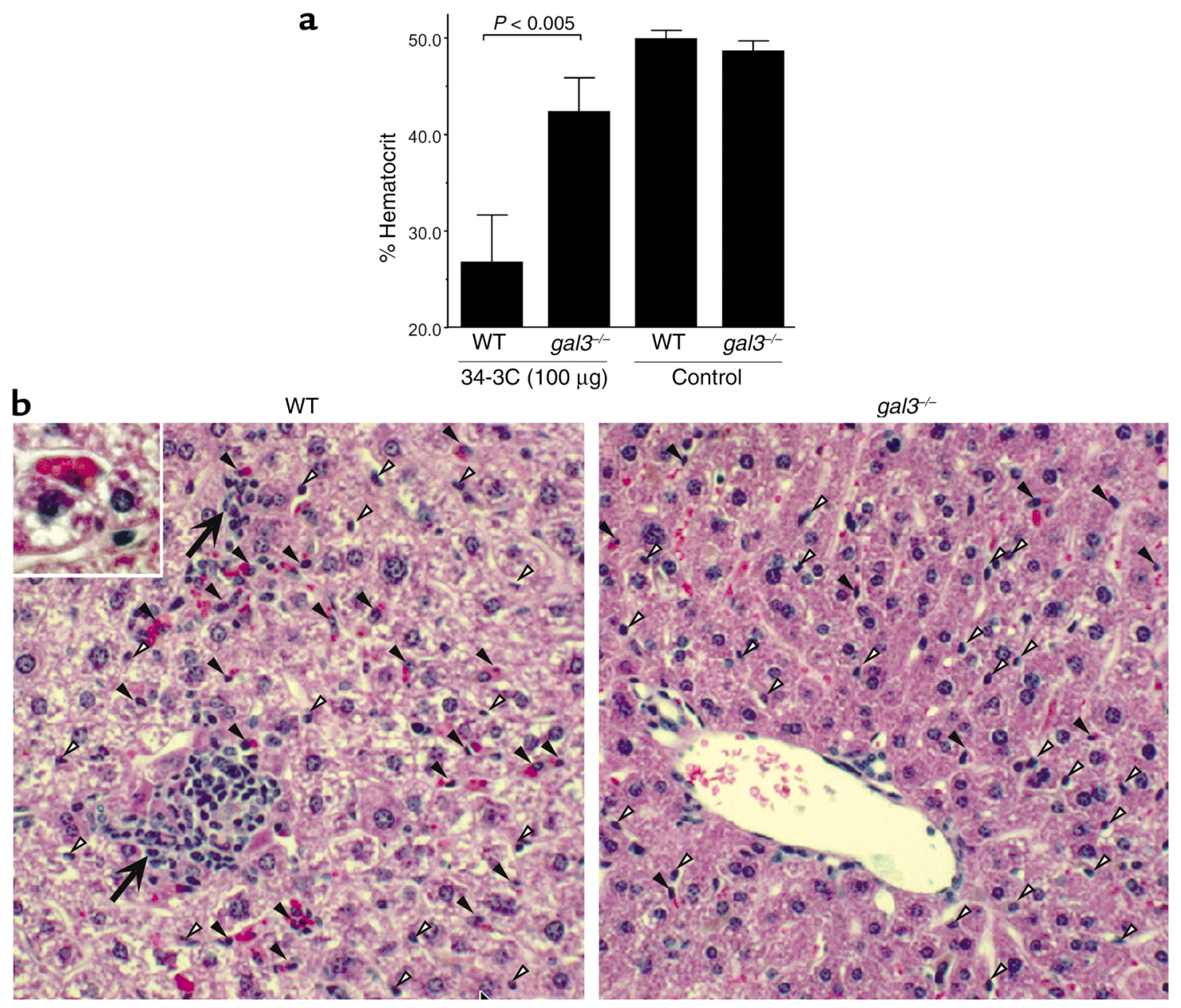

\section{Figure 3}

Impaired IgG-mediated phagocytosis of erythrocytes in gal3-/- mice in vivo. Experimental autoimmune hemolytic anemia was induced by an intraperitoneal injection of the anti-mouse rbc mAb 34-3C. (a) Three days after the injection, hematocrit was measured. The results are the mean \pm SD from four mice of each genotype. (b) Liver sections of wild-type and gal $3^{-/-}$mice were prepared and processed for H\&E staining (original magnification, $\times 400$ ). Open arrowheads point to Kupffer cells that do not contain rbc's and filled arrowheads point to those with phagocytosed rbc's. Arrows point to extramedullary hematopoietic foci. The inset shows a single Kupffer cell demonstrating erythrophagocytosis (original magnification, $\times 1,000$ ). 


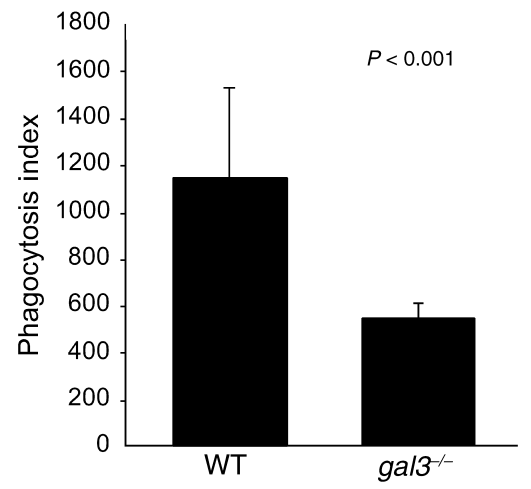

quickly and reached a higher level in wild-type macrophages than in $\mathrm{gal}^{-/-}$macrophages in response to $\mathrm{Fc} \gamma \mathrm{R}$ crosslinkage. Figure 6 is a representative result from four experiments using different cultures of macrophages. The results show that the F-actin ratio in wild-type cells increased by $20.2 \%$ and $27.3 \%$ above the basal level after 5 and 10 minutes of stimulation, respectively, and that after 15 minutes, the ratio began to decline as expected. In contrast, the ratio in $\mathrm{gal3}^{-1-}$ macrophages increased slowly and reached only $2.1 \%$ and $6.8 \%$ above the basal level after 5 and 10 minutes,

\section{Figure 4}

Assessment of the influence of extracellular galectin-3 in phagocytosis of IgG-opsonized erythrocytes. Synchronized phagocytosis assays were performed in cocultures of wild-type and gal3-1macrophages. Data are mean \pm SD from a representative assay 20 minutes after initiation of phagocytosis. Similar results were obtained from three separate experiments.

respectively. These results suggest that galectin-3 functions upstream of actin polymerization.

\section{Discussion}

A major conclusion of this study is that galectin-3 plays an important role in the phagocytic function of macrophages. This is conclusively demonstrated by delayed phagocytosis of opsonized srbc's by gal3-/macrophages compared with wild-type cells. Cellular processes involved in phagocytosis, such as actin polymerization, are also significantly reduced in $\mathrm{gal}^{-1-}$ a
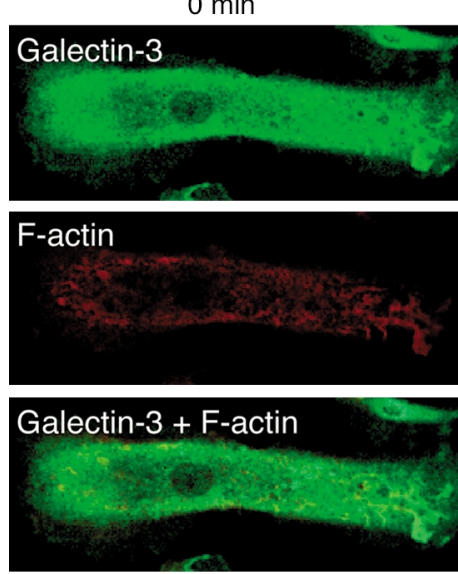

$1 \mathrm{~min}$

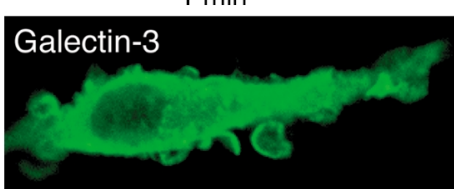

F-actin
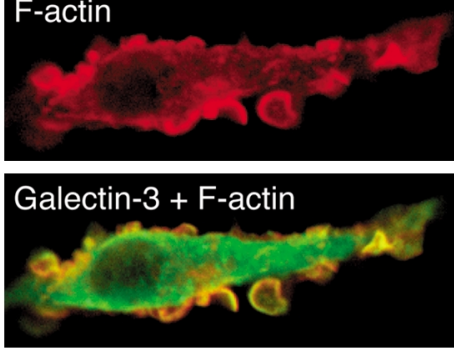

b
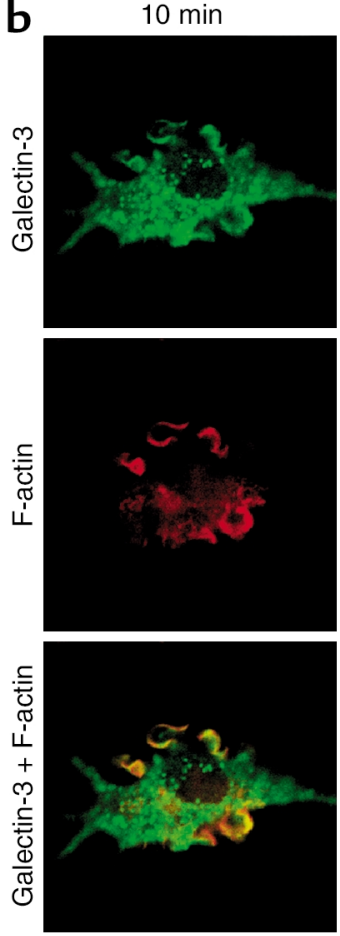

$15 \min$
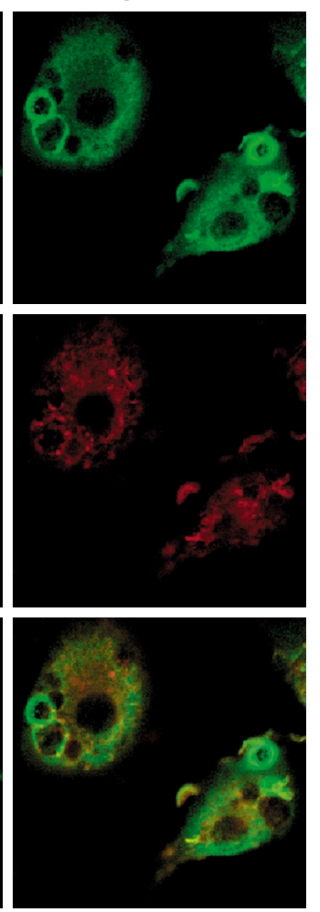

Figure 5

Localization of galectin-3 in actin-rich phagocytic cups and phagosomes. Phagocytosis of srbc's by wild-type and gal3-/- macrophages was performed as described in Figure 1. After the indicated time periods, unbound srbc's were removed and the cells were fixed, permeabilized, and treated with goat anti-galectin-3 Ab, followed by FITC-conjugated mouse anti-goat IgG secondary Ab and rhodamine-phalloidin to stain galectin-3 and actin. (a) Distribution of galectin-3 (upper panels; green) and F-actin (middle panels; red) inside wild-type macrophages. An overlay of these images demonstrates colocalization of F-actin and galectin-3 (lower panels; yellow). Panels on the left show wild-type macrophages before stimulation and those on the right show 1 minute after stimulation with opsonized srbc's. (b) Overlay of galectin-3 and F-actin in wild-type macrophages after 10 and 15 minutes of stimulation. 
macrophages when activated by Fc $\gamma \mathrm{R}$ crosslinkage. Thus, galectin-3 may contribute to the engulfment of particles such as microorganisms and play a critical role in innate immunity. Phagocytosis by macrophages is also an important process in the pathogenesis of Ab-mediated autoimmune diseases. We found that there was a much more pronounced reduction in hematocrit in wild-type mice than in gal3 $3^{-/-}$mice in a mouse autoimmune hemolytic anemia model, suggesting that galectin- 3 contributes to phagocytosis of autoantibody-coated erythrocytes. Therefore, galectin-3 can contribute to the development of these types of diseases. We also found that galectin- 3 is essential for efficient phagocytosis of apoptotic cells in vitro and in vivo. Phagocytosis of apoptotic cells is a prominent feature in many physiological and pathological processes, such as organ development, tissue remodeling, and inflammation (2). Thus, our results suggest that galectin- 3 may be involved in these processes as well.

Galectin- 3 has been shown to bind to the surface of various cell types including erythrocytes through lectin-carbohydrate interactions $(37,38)$. Exogenously added galectin-3 has been shown to activate macrophages in a fashion that is dependent on its lectin properties $(6,15)$. Thus, an obvious explanation for the role of galectin-3 in phagocytosis would be that the secreted lectin binds to the particles to be ingested and then promotes their engulfment by macrophages. However, the addition of lactose to the culture medium, a condition that has been shown to inhibit various extracellular functions of galectin-3, did not inhibit phagocytosis. The results from experiments with cocultures of $\mathrm{gal}^{-/-}$and wild-type macrophages further detract from the probability of a role for extracellular galectin-3 in phagocytosis (Figure 4). Recently, galectin-3 was shown to accumulate in the membrane of mycobacterial phagosomes in Mycobacterium tuberculosis-infected macrophages (39). Galectin-3 continues to accumulate in the phagosomes after macrophages have already acquired mycobacteria and the protein is located at the cytosolic face of the phagosomes. These results suggest that galectin- 3 is sequestered from the cytosol. Similarly, it is possible that the cytosol is a source of the galectin-3 accumulated at the phagocytic cups and phagosomes as observed in the present studies.
How galectin-3 contributes to phagocytosis remains to be elucidated. However, we have obtained significant mechanistic insights by locating this protein in important structures in macrophages undergoing phagocytosis, namely phagocytic cups and phagosomes. Many lines of evidence have indicated that the phagocytic process by various receptors is driven by rearrangement of the actin cytoskeleton and that a variety of signaling molecules can converge at the phagocytic cups and phagosomes, where the actin cytoskeleton is locally reorganized (36). Our finding of distribution of galectin-3 in these structures suggests that galectin-3 may participate in the phagocytic process by controlling the formation of these structures. However, it does not appear that galectin-3 is recruited to the phagocytic cups and phagosomes through direct association with actin, as are many known actin-binding proteins (36). The kinetics of recruitment of galectin-3 to phagosomes, for example, appears to be different from that of actin. At 10 minutes after the initiation of phagocytosis, there was intense staining of actin but weak staining of galectin-3 in phagosomes. On the other hand, at 15 minutes, actin was no longer detectable in the phagosomes, whereas staining of galectin- 3 became intense (Figure 5). In addition, galectin-3 does not contain any of the known actin-binding motifs reported in several actin-binding proteins (40-44).

In summary, galectin-3 plays an important role in the overall function of macrophages. Previous work has shown that this lectin is highly upregulated as monocytes differentiate into macrophages. Other studies have demonstrated that galectin-3, when added to monocytes exogenously, induces superoxide and cytokine production from these cells $(6,35)$. Although direct evidence is lacking, existing information suggests that this lectin should similarly activate macrophages. More recently, it has been shown that galectin-3 is a potent chemoattractant for macrophages (15). Thus, the picture that has emerged is that extracellular galectin-3 contributes to the recruitment and activation of macrophages through a paracrine mechanism. In addition, intracellular galectin-3 contributes to the essential functions of these cells, such as phagocytosis, as demonstrated in this study, as well as cell survival, as demonstrated previously (26). Thus, through a combination of a number of different

\footnotetext{
Figure 6

Reduced actin cytoskeletal rearrangement in ga/3-/- macrophages in response to $\mathrm{Fc} \gamma \mathrm{R}$ stimulation. Wild-type and gal3-/- BMM $\Phi$ were stimulated by Fc $\gamma R$ crosslinkage using specific Ab's for the indicated times. Triton X-100-insoluble F-actin content was measured and expressed as $\mathrm{F}$-actin ratio. Data are representative results of four separate experiments.
}

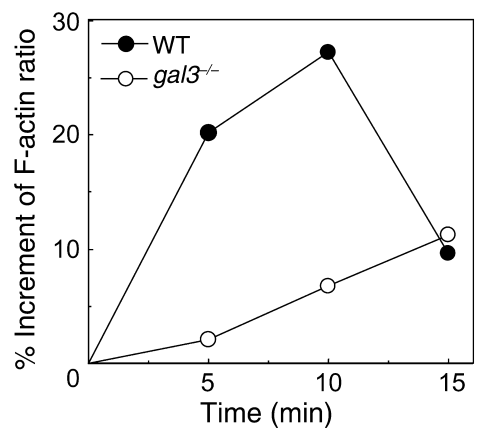


mechanisms, galectin-3 plays important roles in many kinds of biological responses by contributing to the homeostasis and functions of macrophages.

\section{Acknowledgments}

This work was supported by NIH grants RO1 AI-20958, RO1 AI-39620, and PO1 AI-50498 (to F.-T. Liu) and by a grant from the Swiss National Foundation for Scientific Research (to S. Izui).

1. Allen, L.A., and Aderem, A. 1996. Mechanisms of phagocytosis. Curr. Opin. Immunol. 8:36-40.

2. Aderem, A., and Underhill, D.M. 1999. Mechanisms of phagocytosis in macrophages. Annu. Rev. Immunol. 17:593-623.

3. Cooper, D.N. 2002. Galectinomics: finding themes in complexity. Biochim. Biophys. Acta. 1572:209-231.

4. Sato, S., and Hughes, R.C. 1994. Regulation of secretion and surface expression of Mac-2, a galactoside-binding protein of macrophages. J. Biol. Chem. 269:4424-4430.

5. Sato, S., Burdett, I., and Hughes, R.C. 1993. Secretion of the baby hamster kidney $30-\mathrm{kDa}$ galactose-binding lectin from polarized and nonpolarized cells: a pathway independent of the endoplasmic reticulum-Golgi complex. Exp. Cell Res. 207:8-18.

6. Liu, F.-T., et al. 1995. Expression and function of galectin-3, a betagalactoside-binding lectin, in human monocytes and macrophages. Am. J. Pathol. 147:1016-1029.

7. Frigeri, L.G., Zuberi, R.I., and Liu, F.-T. 1993. Epsilon BP, a betagalactoside-binding animal lectin, recognizes IgE receptor $(\mathrm{FC}$ epsilon RI) and activates mast cells. Biochemistry. 32:7644-7649.

8.Zuberi, R.I., Frigeri, L.G., and Liu, F.-T. 1994. Activation of rat basophilic leukemia cells by epsilon BP, an IgE-binding endogenous lectin. Cell. Immunol. 156:1-12.

9. Yamaoka, A., Kuwabara, I., Frigeri, L.G., and Liu, F.-T. 1995. A human lectin, galectin-3 (epsilon bp/Mac-2), stimulates superoxide production by neutrophils. J. Immunol. 154:3479-3487.

10. Hsu, D.K., Hammes, S.R., Kuwabara, I., Greene, W.C., and Liu, F.-T. 1996. Human T lymphotropic virus-1 infection of human T lymphocytes induces expression of the beta-galactose-binding lectin, galectin-3. Am. J. Pathol. 148:1661-1670.

11. Dong, S., and Hughes, R.C. 1996. Galectin-3 stimulates uptake of extracellular $\mathrm{Ca}^{2+}$ in human Jurkat T-cells. FEBS Lett. 395:165-169.

12. Kuwabara, I., and Liu, F.-T. 1996. Galectin-3 promotes adhesion of human neutrophils to laminin. J. Immunol. 156:3939-3944.

13. Inohara, H., Akahani, S., Koths, K., and Raz, A. 1996. Interactions between galectin-3 and Mac-2-binding protein mediate cell-cell adhesion. Cancer Res. 56:4530-4534.

14. Sato, S., and Hughes, R.C. 1992. Binding specificity of a baby hamster kidney lectin for $\mathrm{H}$ type I and II chains, polylactosamine glycans, and appropriately glycosylated forms of laminin and fibronectin. J. Biol. Chem. 267:6983-6990.

15. Sano, H., et al. 2000. Human galectin-3 is a novel chemoattractant for monocytes and macrophages. J. Immunol. 165:2156-2164.

16. Nangia-Makker, P., et al. 2000. Galectin-3 induces endothelial cell morphogenesis and angiogenesis. Am. J. Pathol. 156:899-909.

17. Demetriou, M., Granovsky, M., Quaggin, S., and Dennis, J.W. 2001. Negative regulation of T-cell activation and autoimmunity by Mgat5 N-glycosylation. Nature. 409:733-779.

18. Liu, F.T., Patterson, R.J., and Wang, J.L. 2002. Intracellular functions of galectins. Biochim. Biophys. Acta. 1572:263-273.

19. Dagher, S.F., Wang, J.L., and Patterson, R.J. 1995. Identification of galectin-3 as a factor in pre-mRNA splicing. Proc. Natl. Acad. Sci.U. S. A. 92:1213-1217

20. Kim, H.-R.C., Lin, H.-M., Biliran, H., and Raz, A. 1999. Cell cycle arrest and inhibition of anoikis by galectin-3 in human breast epithelial cells. Cancer Res. 59:4148-4154.

21. Yang, R.-Y., Hsu, D.K., and Liu, F.-T. 1996. Expression of galectin-3 modulates T cell growth and apoptosis. Proc. Natl. Acad. Sci. U. S. A. 93:6737-6742
22. Akahani, S., Nangia-Makker, P., Inohara, H., Kim, H.R.C., and Raz, A. 1997. Galectin-3: a novel antiapoptotic molecule with a functional BH1 (NWGR) domain of Bcl-2 family. Cancer Res. 57:5272-5276.

23. Dietz, A.B., Bulur, P.A., Knutson, G.J., Matasic, R., and Vuk-Pavlovic, S. 2000. Maturation of human monocyte-derived dendritic cells studied by microarray hybridization. Biochem. Biophys. Res. Commun. 275:731-738.

24. Garin, J., et al. 2001. The phagosome proteome: insight into phagosome functions. J. Cell Biol. 152:165-180.

25. Thery, C., et al. 2001. Proteomic analysis of dendritic cell-derived exosomes: a secreted subcellular compartment distinct from apoptotic vesicles. J. Immunol. 166:7309-7318.

26. Hsu, D.K., et al. 2000. Targeted disruption of the galectin-3 gene results in attenuated peritoneal inflammatory responses. Am. J. Pathol. 156:1073-1083.

27. Shibata, T., et al. 1990. Monoclonal anti-erythrocyte autoantibodies derived from NZB mice cause autoimmune hemolytic anemia by two distinct pathogenic mechanisms. Int. Immunol. 2:1133-1141.

28. Fitzer-Attas, C.J., et al. 2000. Fcgamma receptor-mediated phagocytosis in macrophages lacking the Src family tyrosine kinases Hck, Fgr, and Lyn. J. Exp. Med. 191:669-682.

29. Taylor, P.R., et al. 2000. A hierarchical role for classical pathway complement proteins in the clearance of apoptotic cells in vivo. J. Exp. Med. 192:359-366.

30. Fossati-Jimack, L., et al. 2000. Markedly different pathogenicity of four immunoglobulin $\mathrm{G}$ isotype-switch variants of an antierythrocyte autoantibody is based on their capacity to interact in vivo with the low-affinity Fcgamma receptor III. J. Exp. Med. 191:1293-1302.

31. Hazenbos, W.L., et al. 1998. Murine IgG1 complexes trigger immune effector functions predominantly via Fc gamma RIII (CD16). J. Immunol. 161:3026-3032.

32. Frigeri, L., and Apgar, J.R. 1999. The role of actin microfilaments in the down-regulation of the degranulation response in $\mathrm{RBL}-2 \mathrm{H} 3$ mast cells. J. Immunol. 162:2243-2250.

33. Shibata, T., et al. 1990. Monoclonal anti-erythrocyte autoantibodies derived from NZB mice cause autoimmune hemolytic anemia by two distinct pathogenic mechanisms. Int. Immunol. 2:1133-1141.

34. Clynes, R., and Ravetch, J.V. 1995. Cytotoxic antibodies trigger inflammation through $\mathrm{Fc}$ receptors. Immunity. 3:21-26.

35. Jeng, K.C.G., Frigeri, L.G., and Liu, F.-T. 1994. An endogenous lectin, galectin-3 (epsilon BP/Mac-2), potentiates IL-1 production by human monocytes. Immunol. Lett. 42:113-116.

36. May, R.C., and Machesky, L.M. 2001. Phagocytosis and the actin cytoskeleton. J. Cell Sci. 114:1061-1077.

37. Frigeri, L.G., and Liu, F.-T. 1992. Surface expression of functional IgE binding protein, an endogenous lectin, on mast cells and macrophages. J. Immunol. 148:861-869.

38. Feizi, T., et al. 1994. Adhesive specificity of the soluble human lectin, IgE-binding protein, toward lipid-linked oligosaccharides. Presence of the blood group A, B, B-like, and $\mathrm{H}$ monosaccharides confers a binding activity to tetrasaccharide (lacto- $\underline{\mathrm{N}}$-tetraose and lacto- $\underline{\mathrm{N}}$ neo-tetraose) backbones. Biochemistry. 33:6342-6349.

39. Beatty, W.L., Rhoades, E.R., Hsu, D.K., Liu, F.T., and Russell, D.G. 2002. Association of a macrophage galactoside-binding protein with Mycobacterium-containing phagosomes. Cell. Microbiol. 4:167-176.

40. Vandekerckhove, J. 1990. Actin-binding proteins. Curr. Opin. Cell Biol. 2:41-50.

41. Ramesh, N., Anton, I.M., Hartwig, J.H., and Geha, R.S. 1997. WIP, a protein associated with wiskott-aldrich syndrome protein, induces actin polymerization and redistribution in lymphoid cells. Proc. Natl. Acad. Sci. U. S. A. 94:14671-14676.

42. Vardar, D., Buckley, D.A., Frank, B.S., and McKnight, C.J. 1999. NMR structure of an F-actin-binding "headpiece" motif from villin. J. Mol. Biol. 294:1299-1310.

43. Van Troys, M., et al. 1996. The actin binding site of thymosin beta 4 mapped by mutational analysis. EMBO J. 15:201-210.

44. Pope, B., Way, M., Matsudaira, P.T., and Weeds, A. 1994. Characterisation of the F-actin binding domains of villin: classification of F-actin binding proteins into two groups according to their binding sites on actin. FEBS Lett. 338:58-62. 